Western University

Scholarship@Western

Brain and Mind Institute Researchers'

Publications

Brain and Mind Institute

7-25-2015

\title{
Halting the hallmarks: a cellular automaton model of early cancer growth inhibition
}

Jenna Butler

Department of Computer ScienceMiddlesex College University of Western OntarioLondonCanada

Frances Mackay

Department of Applied Mathematics, Middlesex CollegeUniversity of Western OntarioLondonCanada

Colin Denniston

Department of Applied Mathematics, Middlesex CollegeUniversity of Western OntarioLondonCanada

Mark Daley

Department of Computer ScienceMiddlesex College University of Western OntarioLondonCanada

Follow this and additional works at: https://ir.lib.uwo.ca/brainpub

Part of the Neurosciences Commons, and the Psychology Commons

\section{Citation of this paper:}

Butler, Jenna; Mackay, Frances; Denniston, Colin; and Daley, Mark, "Halting the hallmarks: a cellular automaton model of early cancer growth inhibition" (2015). Brain and Mind Institute Researchers' Publications. 150.

https://ir.lib.uwo.ca/brainpub/150 


\title{
Halting the hallmarks: a cellular automaton model of early cancer growth inhibition
}

\author{
Jenna Butler $^{1} \cdot$ Frances Mackay $^{2} \cdot$ Colin Denniston ${ }^{2} \cdot$ Mark Daley $^{1}$
}

Published online: 25 July 2015

(C) Springer Science+Business Media Dordrecht 2015

\begin{abstract}
Cancer treatment is a fragmented and varied process, as "cancer" is really hundreds of different diseases. The "hallmarks of cancer" proposed by Hanahan and Weinberg (Cell 100(1):57-70, 2000) are a framework for viewing cancer within a common set of underlying principles-ten properties that are common to almost all cancers, allowing them to grow uncontrollably and ravage the body. We used a cellular automaton model of tumour growth paired with lattice Boltzmann methods modelling oxygen flow to simulate combination drugs targeted at knocking out pairs of hallmarks. We found that knocking out some pairs of cancer-enabling hallmarks did not prevent tumour formation, while other pairs significantly prevent tumour growth $(p=0.0004$ using Wilcoxon signed-rank adjusted with the Bonferroni correction for multiple comparisons). This is not what would be expected from models of knocking out the hallmarks individually, as many pairs did not have an additive effect but had either no statistically significant effect or a multiplicative one. We
\end{abstract}

NSERC Discovery Grant and Compute Canada.

Jenna Butler

jcamer7@uwo.ca

Frances Mackay

fmackay2@uwo.ca

Colin Denniston

cdennist@uwo.ca

Mark Daley

mark@daleylab.org

1 Department of Computer Science, Middlesex College University of Western Ontario, London, ON, Canada

2 Department of Applied Mathematics, Middlesex College, University of Western Ontario, London, ON, Canada propose that targeting certain pairs of cancer hallmarks, specifically cancers ability to induce blood vessel development paired with another cancer hallmark, could prove an effective cancer treatment option.

Keywords Cancer modelling - Cellular automata . Cancer hallmarks · Lattice Boltzmann · Binary fluid

\section{Introduction}

As of 2004, cancer was the leading cause of death in the developed world and the second leading cause of death in the developing world (World Health Organization 2008), with about 12.7 million cases of cancer in 2008 alone (Ferlay et al. 2008). While much time, money and research are dedicated to cancer the statistics are grim, with little to no progress in some cancers-for example, there has been no significant improvement in survival rates of pancreatic cancer in two decades (Sener et al. 1999). We have created a highly abstract cellular automaton model of early cancer growth and a lattice Boltzmann model of oxygen flow in blood that investigates the impact of knocking out pairs of "cancer hallmarks".

While the traditional reductionist approach to studying cancer has been successful in targeting some forms of the disease, new approaches are needed that can study cancer across scales (Rejniak and Anderson 2012). In silico modelling of cancer is an nascent approach to attacking this problem. Multiscale modelling is a powerful tool for cancer simulation as it allows modelling at the cellular level, and at the fluid level in order to accurately model oxygen flow. Since oxygen availability is critical for cancer progression, modelling both scales provides a more realistic model. Many cancer models currently exist, including: Anderson 
et al.'s multiscale mathematical model of 2-dimensional tumour growth (Anderson et al. 2006); Lloyd et al.'s computational framework for solid tumour growth, which comprised models at the tissue, cellular and subcellular levels (Lloyd et al. 2008); and Ramis-Conde et al.'s hybrid discrete-continuum model which looked at tissue invasion by cancer cells (Ramis-Conde et al. 2008). Models focus on different aspects of tumour growth (including the use of the glycolytic phenotype (Gerlee and Anderson 2008), evolution of cell motility (Gerlee and Anderson 2009) and confined environments (Gevertz et al. 2008)) and employ different modelling approaches [mathematical, (Hirata et al. 2010; Ramis-Conde et al. 2008), hybrid, (Ribba et al. 2004; Gerlee and Anderson 2007), agent-based (Macklin et al. 2012; Sun et al. 2012)]. Readers who wish a broader prospective are directed to the following review articles: (Anderson et al. 2008; Anderson and Quaranta 2008; Rejniak and Anderson 2011).

Currently the state of the art in cancer modelling is spread across different modelling techniques. A recent review paper looking at cancer invasion discusses the use of both hybrid discrete-continuous (HDC) models and immersed boundary models of a cell (IBCell). HDC allows for cells to be modelled discretely but microenvironmental variables such as nutrients and oxygen to be modeled using reaction-diffusion equations. The IBCell model is beneficial for capturing the morphology of a tumour cell as the cells in this model are deformable (Kam et al. 2012). In addition to these two types of agent based models, cellular automaton (CA) models are also used frequently. Gerlee and Anderson created an evolutionary hybrid cellular automaton model where the cancer cells are modelled using cellular automata to capture the behaviour of the tissue as a whole, while using an artificial neural network for cell decisions (Gerlee and Anderson 2007). This type of hybrid cellular automaton model has recently been built on by Shrestha et al. who used a similar model to look at large-scale growth of tumours (Shrestha et al. 2013). Recently, CA models have been used to look at the hallmarks of cancer (Abbott et al. 2006; Basanta et al. 2011; Santos and Monteagudo 2012) as proposed by Hanahana and Weinberg (Hanahan and Weinberg 2000, 2011).

Today survival rates and treatment options for cancers vary widely, largely due to the vast differences among cancers including location, size, aggressiveness, ability to spread and symptoms. Hanahan and Weinberg proposed that almost all cancers actually share eight phenotypic changes and two unique characteristics: SELF SUFFICIENCY IN GROWTH SIGNALS; IGNORING GROWTH INHIBITION; AVOIDANCE OF PROGRAMMED CELL DEATH (APOPTOSIS); LIMITLESS REPRODUCTIVE POTENTIAL; SUSTAINED ANGIOGENESIS; TISSUE INVASION AND METASTASIS; REREGULATED METABOLISM; EVADING THE IMMUNE SYSTEM; INFLAMMATION; and GENETIC INSTABILITY (Hanahan and Weinberg 2000, 2011). The ubiquitous nature of these hallmarks in cancer suggests that treatments able to target them may be useful against multiple types of cancer.

Both Abbott et al. and Santos et al. have developed models looking at these hallmarks (Abbott et al. 2006; Santos and Monteagudo 2012; Monteagudo and Santos 2012, 2013, 2014). Abbott et al. primarily focused on looking at the order in which hallmarks were acquired in the growing tumour. Abbott's results differed from the pathway to cancer proposed by Hanahan and Weinberg, as did the results of an ordinary differential equation model looking at the pathway (Spencer et al. 2004). Abbott's model was an agent based model that simulated the progression of cancer from a single healthy cell to a tumour with at least $90 \%$ cancer cells. They found that hallmarks that confer an advantage to all cells (such as SUSTAINED ANGIOGENESIS which creates blood vessels carrying oxygen into the tumour which all nearby cells can benefit from), do not dominate a cancer clone, whereas hallmarks such as LIMITLESS REPLICATION appear early and dominate as they turn over very quickly.

Santos et al. built on the work of Abbott by using a similar modelling approach, but focused on the impact of removing different hallmarks on tumour growth. They investigated how critical to growth each hallmark was by removing it from the system and comparing the total number of cancerous and healthy cells present with and without the hallmark. They used a cellular automaton model which determined cell division and apoptosis (programmed cell death) based on internal rules and acquired hallmarks. They found that with high mutation rates, the most critical hallmark is APOPTOSIS, while in tumours with little room to grow the IGNORE GROWTH INHIBITION hallmark proved most impactful on overall growth.

We have used model parameters and methods similar to those outlined in Abbott et al.'s work to build upon Santos et al.'s hallmark relevance study. We have implemented five of the six original hallmarks as well as two of the newly introduced hallmarks and enabling characteristics (focusing on those relevant during initial tumour growth), and knocked them out in pairs to see which have the greatest combined effect.

Henderson stated that "in the most general sense, combinations of therapies, whether drugs and/or other modalities, will always play an important role in the management of diseases for which there exists no single specific and totally effective treatment" (Henderson and Samaha 1969). Combination treatment involves pairing multiple treatments with the hope that two in combination will not just be an additive advantage but a multiplicative one. Targeted therapy involves identifying key pathways involved in cancer progression and creating drugs to target these pathways. This model simulates targeted combination 
therapy as we remove key cancer properties (hallmarks) in pairs and compare cancer growth rates to tumours with all hallmarks active. We hypothesize that knocking out pairs of hallmarks will not necessarily have the additive effect of knocking the hallmarks out separately but rather will sometimes have an even greater, potentially multiplicative, combined impact. The following sections include: a brief summary of each hallmark; the event process; pseudocode for the simulation; Lattice-Boltzmann implementation details; parameters used; results and discussion.

\section{Methods}

We have chosen to model two dimensional cancer growth where the biological cells are represented by cellular automata and the oxygen in the environment is modelled as a two-phase fluid using the lattice Boltzman method. Most models in the literature currently restrict themselves to two dimensions as it is more computationally feasible and since cancer does not grow in a sphere but rather an oblate spheroid. Our 2D simulation is easily compared with both existing models and 2D biopsy slices. Here we will present a high level outline of the method, and each section will be covered in more detail below. Pseudocode describing the simulation is provided in Table 2 . The simulation begins with a single healthy cell at the center of a 2-dimensional grid. An event queue keeps track of cellular events, and initially a single mitotic event is placed on the queue for the healthy cell. Each event popped from the queue is another loop in the model and puts that cell through a life cycle. The cell is checked for whether it still has enough oxygen to survive, is in a location with growth factor, has access to blood, has space to grow, and has sufficiently long telomeres. If all of these checks are successful, or if mutations confer these abilities, the cell enters a mitotic event. This creates a daughter cell and potentially introduces mutations into the daughter or parent. Both cells have events scheduled for some point in the future and are added to the event queue, then the next event is popped. Oxygen is consumed by cells when they divide or every 25 time steps if they are not actively dividing. The following sections include: a brief summary of each hallmark; the event process; pseudocode for the simulation; LatticeBoltzmann implementation details; parameters used; results and discussion.

\subsection{Modelling the hallmarks}

The "hallmarks of cancer" proposed by Hanahan and Weinberg are changes to cell phenotype (characteristics of the cell based on its genotype and the environment-in our model, phenotype is the collection of hallmark mutations a cell has along with parameter values) that seem to be consistent across a variety of cancers (Hanahan and Weinberg 2000, 2011). These hallmarks give a structure and common signature to a disease that is actually a combination of hundreds of different types of diseases. We have simulated 5 of the 6 original hallmarks and two of the recently added characteristics and hallmarks that were described in (Hanahan and Weinberg 2011). This model is specifically focused on the pre-metastatic growth, when a patient has the greatest chances of survival. Therefore, we have not modelled the sixth hallmark, tissue invasion and metastasis. To keep our results comparable with previous work in this field, we have not included inflammation or energy metabolism in our model. Our model is inspired by work in artificial life where agent based and mathematical models have been used to simulate cancer growth and angiogenesis (Maley and Forrest 2000; Bentley et al. 2008). Here we will briefly describe each hallmark put forth by Hanahan and Weinberg that we are including in this model, as well as their implementation. At the end of this section pseudo code describing the cell lifecycle is included.

\subsection{Self-sufficiency in growth signals-hallmark symbol: SG}

Normally, cells are generally in a quiescent state where they are functioning but not actively proliferating. In order for a cell to become mitotically active, it needs mitogenic growth signals, which stimulate it to move from the quiescent state to a dividing one. Normal, healthy tissues regulate the creation and release of growth signals very carefully, balancing the number of actively dividing cells so no area is overburdened with resource requests. Cancer cells de-regulate these signals. In fact, self-sufficiency in growth signals is said to be the most fundamental trait of cancer cells (Hanahan and Weinberg 2011).

The deregulation of these signals in cancer is fairly well understood (Witsch et al. 2010; Perona 2006). Cancer cells can sustain their growth through a variety of mechanisms including:

- sending growth signals to surrounding cells which reciprocate by sending even more growth factors;

- increasing their own cell-surface receptors for growth signals so that normal levels of signal can have an enhanced effect;

- activation of growth pathways downstream of signal reception, which enhances proliferation but also reduces their dependency on external stimuli;

- interference with negative feedback loops which normally lessen signals and therefore assist in maintaining homoeostasis. Hanahan and Weinberg have predicted that "compromised negative-feedback loops" will be 
found to be "wide spread among human cancer cells" (Hanahan and Weinberg 2011). They also believe cancer cells may use this ability to disturb feedback loops to gain resistance to drugs which target cell cycle signalling.

It is hypothesized by Hanahan and Weinberg that cells may have built-in safety mechanisms to avoid excessive growth, and they postulate that cancer may be involved in a delicate trade-off between increased signalling for fast growth and not growing so fast as to trigger interference from safety mechanisms (Hanahan and Weinberg 2011). It is also possible that cancer cells could somehow be deactivating these safety mechanisms.

In order to model this hallmark, we have programmed healthy cells such that they are only able to divide within a certain area of the total growth environment. Healthy cells can only divide up to some predefined limit, which is akin to an area with growth factor present. In certain areas (outside the limit) there is not enough growth factor to signal proliferation, and therefore healthy cells become non-proliferative in this area. Cancer cells however can obtain the SUSTAINED GROWTH (SG) mutation, allowing them to actively divide outside of the predefined boundary, modelling the ability to initiate growth themselves via a variety of mechanisms.

\subsection{Ignore growth inhibition-hallmark symbol: IGI}

Coupled with a tissue's tight regulation over growth inducing mechanisms, tissues also have strong negative regulation over cell growth, actively suppressing it as opposed to passively not taking part due to the absence of growth promoting factors. The genes which do this are often referred to as tumour suppressor genes as they can actively limit cell growth and proliferation through a variety of mechanisms including stopping the cell cycle and cell-cell contact inhibition.
While there are a variety of ways cell growth can be negatively controlled, we have chosen to look at the impact of pressure exerted on cells by other cells. When cells grow together the cell-to-cell contact has an inhibitory effect on growth. This mechanism, called contact inhibition, is often turned off or perhaps ignored in many cancer cells. An example of a gene involved in this pathway is the NF2 gene, the loss of which triggers human neurofibromatosis, a disease which deposits tumours throughout a patient's body. The gene product of NF2, Merlin, strengths cell-cell adhesion and also sequesters growth factors, limiting cell growth.

In order to model cancer's ability to ignore anti-growth signals, we have implemented an empty space requirement for growth. This models one of cancer's anti-growth avenues-avoid contact inhibition. In this model, healthy cells stop actively growing once there is no more space available on the lattice. Cancer cells in our system can have the IGNORE GROWTH INHIBITION (IGI) hallmark activated which allows cells to grow even without space (thereby modelling the impact of mutations which cause a cell to ignore contact inhibition). These cells have a "competition" factor (c). If a cell with the IGI hallmark attempts to grow and is out of space, they compete with cells around them and can potentially take over the space another cell is occupying in order to grow. Each time a cell competes (done once every attempt to divide without space), the cell has a $1 / c$ likelihood of successfully gaining the space, where $c$ is the competition factor mentioned in Table 1.

\subsection{Resisting cell death-Hallmark symbol: IA}

Apoptosis, or programmed cell death, is a normal process in animal tissue. Apoptosis assists in keeping the balance of healthy living cells with dead or dying cells, keeping cell populations in check and dealing with damaged or old cells. In addition to helping maintain balance, it is also a

Table 1 Parameters used in simulations

\begin{tabular}{llll}
\hline Description & Symbol & Value & References \\
\hline Initial telomere length & $t$ & 100 & Abbott et al. (2006) \\
Evade apoptosis factor & $e v$ & 10 & Abbott et al. (2006) \\
Mutation rate & $\mathrm{m}$ & 500 & Chosen to lay between two used in Santos and Monteagudo (2012) \\
Random death rate & $\mathrm{d}$ & 10000 & Simulation \\
Competition likelihood & $c$ & 10 & Abbott et al. (2006) \\
Angiogenesis immunity & $a i$ & 10 & Simulation \\
Avoid immunity & aip & 10 & Simulation \\
Immunity death & $i$ & 1000 & Simulation [equal to random cell death in Santos and Monteagudo (2012)] \\
Genetic instability factor & gif & 10 & Simulation \\
Blood density & $\rho_{B}$ & $300 \mathrm{~mol} / \mathrm{m}^{3}$ & Erbertseder et al. (2012) \\
Boundary oxygen density & $\rho_{O}$ & $9 \mathrm{~mol} / \mathrm{m}^{3}$ & McArdle et al. (2005) \\
Thermal diffusivity & $D$ & $0.134 \mathrm{~mm}^{2} \mathrm{~s}^{-1}$ & Valvano et al. (1985) \\
\hline
\end{tabular}


safety mechanism which prevents damaged cells from passing on damage (Lowe et al. 2004; Evan and Littlewood 1998).

Apoptosis has been found to be triggered by different events, many of which are common during the progression from normal, healthy cell to cancerous cell, such as high levels of oncogene expression and DNA damage. Although it is known that DNA damage and oncogene overexpression can lead to cancer, which should trigger apoptosis, it has also been found that cancer cells sometimes manage to avoid apoptosis (Hanahan and Weinberg 2011).

Various cell conditions leading to apoptosis have been identified, with one of the most notable being DNA damage (Junttila and Evan 2009). Major DNA breaks or chromosomal abnormalities are sensed by tumour-suppressor protein 53 (p53, encoded in gene TP53) which can in turn activate apoptosis if the damage cannot be fixed. While cancer cells evolve many mechanisms to avoid apoptosis, one of the most common is loss of TP53 function (found to be gone in $50 \%$ of all cancers). This allows a cell to build up DNA damage unchecked which can lead to additional mutations and passing down of damaged DNA.

We have chosen to model the apoptotic pathway primarily as a sensor for DNA damage. Once a healthy cell has sustained any mutations, it is possible for it to enter the apoptotic state (simulating the cell being killed by apoptosis). Since apoptosis can be activated due to genetic damage, the chances of a cancer cell dying via apoptosis increases with each subsequent mutation (in our model). A cell has a $m / a$ probability of being killed by this mechanism, where $m$ is the number of mutations already sustained, and $a$ is an apoptosis chance variable contained in each cell (see Table 1 for exact parameters). Cells with a mutation in this mechanism cannot die by apoptosis, regardless of the number of mutations acquired. This AVOIDS APOPTOSIS mutation is referred to as AA throughout the paper.

\subsection{Enabling replicative immortality-Hallmark symbol: IT}

Most normal healthy cells have a limit to how many times they can divide before they enter a viable but non-proliferative state called senescence. Healthy cells that manage to avoid senescence often instead enter a crisis state, ending with cell death. In contrast, cancer cells seem to require unlimited replication to grow into a tumour of macroscopic size. When cells are propagated in culture, leading to senescence, and then for some to crisis, many of the cells die. At this point it occasionally happens that a cell line comes up displaying this unlimited replication, effectively becoming immortal, continuing to grow without hitting senescence or crisis (Hanahan and Weinberg 2000, 2011).

It is believed cells have a feature which only allows them a certain number of replications-this feature is the telomere. Telomeres are segments of DNA made up of multiple repeating 6-nucleotide segments capping the ends of chromosomes. DNA replication is not a perfect process and always results in the loss of some material at the ends of chromosomes. Telomeres protect the "necessary" DNA by themselves being shortened at each replication. Eventually however they become too small to effectively protect the DNA and at this point it is believe senescence can be triggered.

Telomerase is a DNA polymerase that builds these telomeres. Normally it is not active in healthy cells, however it is found to be turned on in $90 \%$ of suddenly immortal cells (Hanahan and Weinberg 2011). Telomerase then can continuously extend the ends of DNA making it so they never reach a size small enough to trigger senescence or crisis. Accordingly, the presence of telomerase is correlated with resistance to both of these fates. It is believed both of these events (senescence and crisis) are natural barriers to cancer. Rogue cells may develop mutations, be growing out of control of the body's signalling, and begin rapid division. These cells can be abruptly stopped when their full replicative potential is reached, causing them to not be able to divide further and not make it to a macroscopic tumour. Cells which manage to activate telomerase however keep their telomeres long enough to avoid senescence and crisis and therefore forever pass on their mutations. As such, telomere shortening is thought to be one of the barriers cancer cells must defeat to progress into a dangerous tumour (Hanahan and Weinberg 2011). One particular example supporting this hypothesis is the work of Artandi and DePinho who found that mice genetically predisposed to certain cancers had weakened tumourigenesis when born without telomerase (Artandi and DePinho 2010).

We have included in our model the ability for a cell to become immortal. Every cell is equipped with a "telomere" variable that decreases by one with every cell division. This variable limits healthy cells to 100 cell divisions (Abbott et al. 2006). Cells can acquire the IGNORE TELOMERE hallmark which allows them to effectively ignore this limitation and not be bound by their telomeres. This replicates the biological activity of telomerase which continues increasing telomeres after they are shortened, preventing their length from impeding growth. Cells with the IGNORE TELOMERES (IT) hallmark activated can divide 
forever, regardless of telomere length, so long as all other required conditions are met (oxygen, space, etc).

\subsection{Inducing angiogenesis-Hallmark symbol: A}

Vasculature, the system of blood vessels in the body, serves two major purposes for cell groups: delivering nutrients and oxygen, and removing waste products and carbon dioxide. Both healthy and cancerous cells depend on and need this system. Typically, vasculature is quite stable. It is originally developed during embryogenesis, when the processes of vasculogenesis (the birth of new endothelial cells and their development into tubes) and angiogenesis (sprouting) occur. After embryogenesis is complete, angiogenesis is only turned on transiently during wound healing and as part of the female reproductive cycle. A key early development in tumour growth is the activation of this normally quiescent angiogenic process, causing new vasculature to sprout towards and even into tumours (Hanahan and Weinberg 2000, 2011). This is dangerous as it not only provides the tumour with fresh oxygen, nutrients, and waste removal, but also gives it a system to use to travel through the body.

When angiogenesis is induced in tumours it often results in poorly set up vasculature with issues such as leakiness, erratic blood flow and excessive and convoluted branching. While this process was previously thought to occur later in tumour development, such as once the tumour was rapidly growing and macroscopic in size, research in the past two decades has found it can begin as early as the pre-malignant microscopic stage of growth (Raica et al. 2009).

Tumours exhibit widely varied tumour-induced vasculature, even within the same organ. For example, adenocarcinoma of the pancreatic ducts is hypovascularized (Olive et al. 2009), while pancreatic neuroendocrine carcinomas can be densely vascularized (Detjen et al. 2010). The variety of tumour induced angiogenesis seen suggests that angiogenesis is initially switched on, but complexly regulated and impacted throughout tumour growth. While the mechanism of angiogenesis switch activation can vary, the net result is a common inductive signal. In some cases oncogenes activate angiogenesis. These also can stimulate proliferation which means other unique hallmarks (such as sustained growth) can possibly be activated by the same rogue players.

Since it is believed angiogenesis is at least initially switched on in tumour growth, and this model aims to simulate early tumour growth, we have included this hallmark. In the simulation, angiogenesis is modelled at a very basic level. Cells that have the INDUCE ANGIOGENESIS (A) hallmark activated are thought to be on the path of new vasculature. In the simulation, every lattice location has an associated oxygen value that changes over time based on consumption and supply, and is modelled using Lattice Boltzmann methods (LBM) (full implementation details on this model are described in Sect. 3). At each division a cell checks if it has enough oxygen to survive by checking with the lattice Boltzmann simulation to determine how much oxygen is present where it is located. If a cell does not have enough oxygen it either becomes quiescent or dies (depending on how much oxygen is present). However, if it is on the path of vasculature because it is inducing angiogenesis, it is getting oxygen directly from the vasculature so it does not require enough oxygen in its lattice cell as calculated by the LBM. Neighbours of cells on the new vasculature also benefit from this via diffusion from the vasculature and are able to survive in places where there would not otherwise be enough oxygen provided by the original healthy vasculature.

In this model angiogenic cells also have a higher chance of being killed by the immune system, as immune cells travel via the vasculature. Angiogenic cells and their neighbours have a $(a i) *(1 / i)$ chance of being killed by the immune system at each replication step, where (ai) is the angiogenesis immunity parameters and $i$ is the immune death probability parameter (Table 1).

\subsection{Genetic instability_Hallmark symbol: GU}

Cancer cells acquire the above mentioned hallmarks in large part because of successive changes to the genome of neoplastic cells (Hanahan and Weinberg 2011). Some mutational changes will confer an advantage to the cell or cells, allowing them to grow and dominate in an environment. Therefore, the many steps from normal cell to cancerous cell, and the subsequent stages of tumour growth, can be viewed as the successive accumulation of favourable chance mutations each creating a new cell clone group. However, not all clonal expansions need be caused by mutations, as research has shown that epigenetic changes can also impact gene expression (Berdasco and Esteller 2010; Jones and Baylin 2007).

Many innate cell systems are able to detect and repair DNA damage; consequently, the number of spontaneous mutations in a cell generation tends to be low. Cancer cells however often have higher than normal mutation rates, which can be achieved in a variety of ways. There can be an increased sensitivity to mutagenic agents or breakdown in any of the genetic maintenance machinery or pathways. Also, a disturbance in the cell machinery that detects and fixes mutations (for example, TP53, mentioned earlier) can lead to an increased mutation rate. The process of detecting 
and fixing DNA damage is complex, and so has a lot of potential places breakdowns can happen, such as:

- Machinery that detects damage and activates repair pathways;

- Machinery that repairs DNA;

- Machinery that kills a cell if too much damage is acquired;

- The pathways involved with the inactivation and interception of mutagenic molecules.

Other cell components can be included in the list of "caretakers" that watch over the genome. Some research found that there may even be certain areas of the genome where aberrations are likely to lead to neoplastic growth, an interesting observation made after seeing recurrence of specific amplifications and deletions at certain sites in breast cancer (Korkola and Gray 2010).

Currently it is known that the genetic changes in cancer vary across tumours, tissues and types, however one thing that is agreed upon is the vast array of cell maintenance and repair mechanisms that can be damaged as well as the prevalence of copy number changes in cancers. These widespread changes point to genome instability as an enabling characteristic of cancer in general, and possibly one that is causing the acquisition of cancer hallmarks (Hanahan and Weinberg 2011).

Working with this belief, that genome instability could cause cells to acquire some hallmarks, we have added it as an enabling characteristic in this cancer simulation. While there are many different pathways and mechanisms that can be impacted in cancer growth and cause genetic instability, the end result is a genetically unstable cell which has a higher likelihood of mutation. We model this characteristic with the GENETICALLY UNSTABLE hallmark (GU) which when active increases the chances of mutation in each mitotic event by a factor of ( $1 /$ gif $)$ where gif is the genetic instability factor parameter (Table 1). Modelling it in this way allows for hallmarks to still be acquired spontaneously due to any number of factors, but also allows cells to accumulate mutations more quickly if they are genetically unstable.

\subsection{Evading immune destruction-Hallmark symbol: AI}

Listed as an "emerging hallmark" in the updated cancer hallmarks paper, the ability for cancer to seemingly evade destruction by the immune system is an unresolved issue that appears to play a large role in cancer growth (Hanahan and Weinberg 2011). It has long been believed that the immune system is like a constant surveillance system, watching the body for signs of foreign cells or incipient cancer cells, eradicating them if they are found. By this logic, cells that manage to grow into full macroscopic tumours must have somehow avoided detection or destruction by that same system.

One piece of evidence in the argument that the immune system is involved in the early detection and eradication of cancer cells is the fact that individuals who are immunocompromised have a much higher incidence of certain cancers (Vajdic and Leeuwen 2009). However many of these are cancers caused by viruses, and so it may seem that perhaps the role of the immune system in cancer prevention is just minimizing the viral load on a body. Recently however, some studies have shown that even in non-virus-induced cancer, the immune system still plays a significant role as a barrier to cancer progression (Hanahan and Weinberg 2011).

Studies with genetically engineered mice back up this claim. Mice that have been engineered to lack various pieces of the immune system had an increased incidence of tumour formation. Mice that were engineered to be deficient in multiple pieces of the immune system had even higher rates of cancer (Teng et al. 2008; Kim et al. 2007).

Hanahan and Weinberg believe there is some evidence of antitumour immunity but that it has yet to be proven ubiquitously enough to be considered a core hallmark and as such have set it as an "emerging hallmark". We have chosen to include emerging hallmark AI in this study, as we are interested in a high-level, abstract model. We could assume this general model is of a type of solid mass tumour that is affected negatively by the immune system, as many cancers have been shown to be. This simulation has a very simple, basic model of immune system surveillance. In the model, cells which have sustained mutations have the possibility of being killed by the immune system at every life cycle (a probability of $1 / i$ where $i$ is the immune death parameter in Table 1). We then model cancer's ability to possibly avoid this with the AVOIDS IMMUNE SYSTEM hallmark (AI) which, when activated, lowers the probability of a cancer cell being killed by the immune system by a factor of aip, the avoids immune system parameter (Table 1).

\subsection{A note on hallmarks not included}

This model specifically includes 5 of the original 6 hallmarks of cancer, one of the two emerging hallmarks, and one of the two enabling characteristics. The choice of what to model was informed by the scope and type of study, the biological relevance at the early growth phase, and the 
computational and modelling feasibility. Those interested in learning more about the hallmarks not included (or any hallmarks) are directed to the revised Hallmark paper, Hallmarks of cancer: The next generation (Hanahan and Weinberg 2011) and the papers referenced therein.

Firstly, we left out the "tissue invasion and metastasis" hallmark from the original hallmark paper (Hanahan and Weinberg 2000). This particular cancer simulation is focused on the early, pre-metastatic growth phase of cancer. We are interested in early growth, the appearance of the hallmarks, the time when tumours reach visible size and what hallmark-stopping efforts are most useful at this time. Patients whose tumours are found before they spread have a much better chance of survival and we are interested in this early phase of growth. This simulation looks specifically at early tumour growth and the development of the primary tumour, and as such does not include invasion or metastasis in the simulation.

The enabling characteristic we did not include is "tumour promoting inflammation". Hanahan and Weinberg point out that inflammation is sometimes present at very early stages of neoplastic progression, and has been found to be clearly capable of assisting in the progression from incipient neoplasias to fully functioning cancer tumours. Also, some inflammatory cells release reactive oxygen species which are mutagenic to the surrounding cells, possibly assisting these cells in gaining advantageous mutations. As such, Hanahan and Weinberg have classified inflammation as an "enabling characteristic" of cancer, however they stated that of the two enabling characteristics, the most prominent is genomic instability. We have chosen not to include this characteristic in our simulation. We are modelling early cell growth, which has been shown to sometimes have inflammation present, but not always. Also, the role of inflammation is still largely unknown. Many of the cell types that lead to inflammation, such as cells of the innate immune system, play a dual role of assisting in cancer development and trying to stop it. Inflammation appears to be involved in a host of developments, specific to what cells are present, the microenvironment, etc. As such, there is no obvious single impact on early tumour growth that can be abstracted to a parameter that could be modelled in the type of high level model we are interested in studying. In the future adding a microenvironment at a lower level of abstraction would be beneficial and would allow the addition of some inflammatory responses.

Lastly we have not modelled the emerging hallmark of "deregulated cellular energetics". This potential hallmark involves a cancer cell's ability to change their energy metabolism pathway from the high efficiency aerobic respiration, to the lower efficiency anaerobic respiration. As this is an emerging hallmark, Hanahan and Weinberg have not decided that it is common enough to be a full hallmark. Also, this high level model is only looking at one aspect of the tumour microenvironment-the impact of oxygen availability on the hallmarks. We are not specifically modelling ATP creation or glucose, and as such we have not included this emerging hallmark.

\subsection{Event queue}

Mitotic events are the driving force in this model. An event queue keeps track of all events scheduled for the simulation. Initially, the cell has a mitotic event scheduled for 511 time steps in the future. When the event is popped the time is checked. If the time for the event is beyond the current time in the simulator the current time is updated. If the cell is to grow in a North, East, South or West direction, the time is scheduled 5-11 time points in the future (chosen by random number from a uniform distribution). If the cell is growing on a diagonal on the grid then the event is scheduled for $7-14$ ( $\sqrt{2}$ of the normal mitotic time) to account for the increase in spatial distance. 


\subsection{Lifecycle psuedocode}

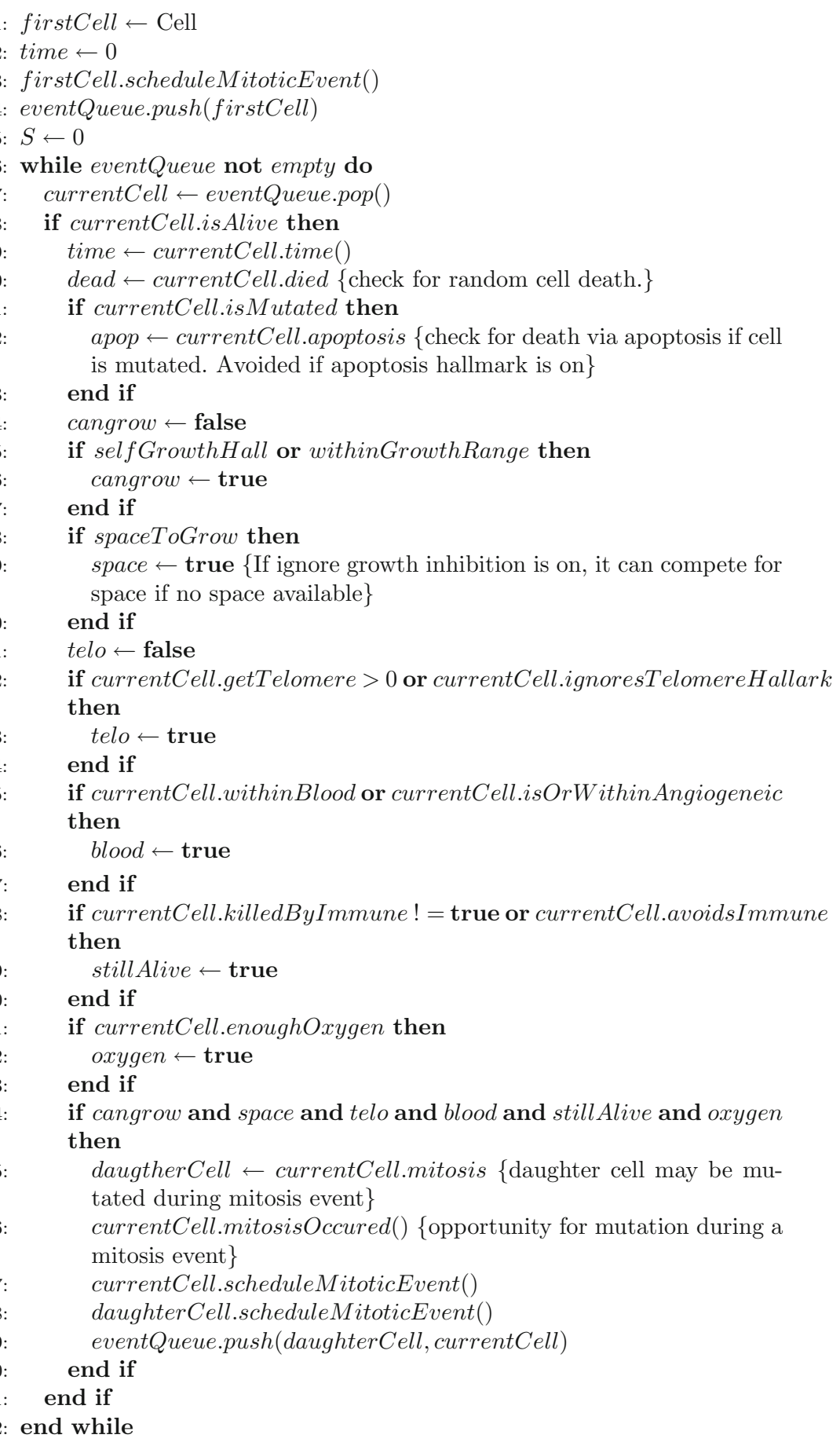

\section{Lattice-Boltzmann implementation details}

Lattice-Boltzmann algorithms (Chen and Doolen 1998; Succi 2001), have become increasingly popular as methods used to model coarse-grained fluid dynamics. These methods use a discretization of time, space, as well as velocity in order to solve for the motion of a set of partial distribution functions, $f_{i}(\mathbf{x}, t)$, each corresponding to a discrete velocity vector, $\mathbf{e}_{i}$, which evolve according to a discretized version of the linearized Boltzmann equation. Macroscopic fluid quantities are then determined via moments of these distribution functions.

In order to model the transport of oxygen dissolved in blood, we use a two component lattice-Boltzmann algorithm. Here, the quantities of interest are the total density $\rho=\rho_{B}+\rho_{O}$ which should satisfy both the continuity and 
Navier-Stokes equations, and the density difference between the two components $\phi=\rho_{O}-\rho_{B}$, which will evolve according to a convection-diffusion equation. In these expressions, $\rho_{O}$ corresponds to the density of oxygen in the blood, and $\rho_{B}$ is the density of the remaining blood constituents. In order to model these two quantities we follow the binary fluid approach of (Orlandini et al. 1995; Swift et al. 1996), and introduce two sets of distribution functions, $f_{i}$ and $g_{i}$, whose moments correspond to the physical variables,

$$
\begin{aligned}
& \rho=\sum_{i} f_{i} \\
& \rho u_{\alpha}=\sum_{i} f_{i} e_{i \alpha} \\
& \phi=\sum_{i} g_{i},
\end{aligned}
$$

where $\mathbf{u}$ is the local fluid velocity. The time evolution of these distribution functions is governed by the following discretized Boltzmann equations,

$$
\begin{aligned}
f_{i}\left(\mathbf{x}+\mathbf{e}_{i} \Delta t, t+\Delta t\right)-f_{i}(\mathbf{x}, t)= & -\frac{\Delta t}{\tau_{\rho}}\left(f_{i}(\mathbf{x}, t)-f_{i}^{e q}(\mathbf{x}, t)\right) \\
g_{i}\left(\mathbf{x}+\mathbf{e}_{i} \Delta t, t+\Delta t\right)-g_{i}(\mathbf{x}, t)= & -\frac{\Delta t}{\tau_{\phi}}\left(g_{i}(\mathbf{x}, t)-g_{i}^{e q}(\mathbf{x}, t)\right) \\
& +h_{i}(\mathbf{x}, t) \Delta t
\end{aligned}
$$

The first term on the right hand side describes a single time relaxation towards the equilibrium distribution functions, $f_{i}^{e q}$ and $g_{i}^{e q}$ (Bhatnagar et al. 1954), while $h_{i}$ is a forcing term we have introduced in order to remove oxygen locally from the system when it is consumed by the cancer cells. To model these equations we use a nine velocity, 2D algorithm with velocity vectors,

$\mathbf{e}_{i}=\left[(0,0),\left( \pm v_{c}, 0\right),\left(0, \pm v_{c}\right),\left( \pm v_{c}, \pm v_{c}\right)\right]$,

where $v_{c}=\Delta x / \Delta t$, with $\Delta x$ and $\Delta t$ corresponding to the lattice spacing, and timestep respectively.

In order to satisfy conservation of mass and momentum we choose the equilibrium distribution functions according to,

$$
\begin{aligned}
& \sum_{i} f_{i}^{e q}=\rho \\
& \sum_{i} f_{i}^{e q} e_{i \alpha}=\rho u_{\alpha} \\
& \sum_{i} g_{i}^{e q}=\phi \\
& \sum_{i} g_{i}^{e q} e_{i \alpha}=\phi u_{\alpha},
\end{aligned}
$$

and define the higher moments and forcing term by the following equations,

$$
\begin{aligned}
& \sum_{i} f_{i}^{e q} e_{i \alpha} e_{i \beta}=P_{\alpha \beta}+\rho u_{\alpha} u_{\beta} \\
& \sum_{i} g_{i}^{e q} e_{i \alpha} e_{i \beta} \\
& \sum_{i} h_{i}=F \\
& \sum_{i} h_{i} e_{i \alpha}=0 .
\end{aligned}
$$

Here $P_{\alpha \beta}$ is the pressure tensor, $\Gamma$ is the mobility, which is related to the diffusion constant, $\mu$ is the chemical potential difference between the fluid components, and $F$ is an oxygen sink term describing the amount by which $\phi$ changes at a given timestep. With these choices, a Chapman-Enskog expansion of our Boltzmann equations [Eq. (3)] can be shown to reproduce the continuity and Navier-Stokes equations,

$\partial_{t} \rho+\partial_{\alpha} \rho u_{\alpha}=0$

$\rho \partial_{t} u_{\alpha}+\rho u_{\beta} \partial_{\beta} u_{\alpha}=-\partial_{\beta} P_{\alpha \beta}+\eta \nabla^{2} u_{\alpha}$,

as well as a convection-diffusion equation with oxygen sink term, $F$,

$\partial_{t} \phi+\partial_{\alpha}\left(\phi u_{\alpha}\right)=\left(\tau_{\phi}-\Delta t / 2\right)\left[\Gamma \nabla^{2} \mu-\partial_{\alpha}\left(\frac{\phi}{\rho} \partial_{\beta} P_{\alpha \beta}\right)\right]+F$.

to second order in the derivatives. Here, the viscosity, $\eta$, is defined according to $\rho\left(\tau_{\rho}-\Delta t / 2\right) v_{c}^{2} / 3$. For the pressure, $P_{\alpha \beta}$, and chemical potential difference, $\mu$, we use the equations given in (Orlandini et al. 1995; Swift et al. 1996), which were derived based on a free energy description of the fluid mixture,

$\mu=-\frac{\lambda}{2} \frac{\phi}{\rho}+\frac{\xi}{2} \ln \left(\frac{\rho+\phi}{\rho-\phi}\right)$

$P_{\alpha \beta}=[\rho \xi+\phi \mu] \delta_{\alpha \beta}$.

Here $\lambda$ and $\xi$ are parameters determining the state of the system; for $\xi<\lambda / 2$ phase separation of the two components occurs. We therefore always work in a regime where $\xi>\lambda / 2$ and the oxygen remains mixed in the blood. In this framework, the diffusion constant for the model, $D$, is given by,

$D=\frac{\left(\tau_{\phi}-\Delta t / 2\right) \Gamma \lambda}{2 \rho}$.

For the lattice-Boltzmann algorithm, we use a density of $\rho_{B}=300 \mathrm{~mol} / \mathrm{m}^{3}$ (Erbertseder et al. 2012) throughout the simulation domain, and set the density of oxygen to $\rho_{O}=$ $9 \mathrm{~mol} / \mathrm{m}^{3}$ at the boundary, representing a continual supply of oxygen to the system. We choose a diffusion constant, $D=0.134 \mathrm{~mm}^{2} / s$ (Valvano et al. 1985), corresponding to 
the thermal diffusivity of colon cancer. The oxygen grid is updated every 25 time steps (time in the cellular automata model) and each cell at that point consumes oxygen if it has not consumed already during a mitotic event. The consumption level for normal cancerous cells is 0.019 . The lattice Boltzmann grid is finer than the CA grid (a ration of 1 cancer cell to 9 lattice Boltzmann cells), and so at each time step of the cancer cells, each cancer cell calculates the amount of oxygen present in all lattice Boltzmann cells mapped to it and gets a total oxygen value. These are dimensionless and are parameters chosen by fixing the consumption and diffusion rates, and iteratively determining what requirements gave the most physiologically relevant results.

\subsection{Parameter values}

Parameters (Table 1) for the model were either chosen from the literature or by searches of the parameter space. Parameters used in the models by Abbott (Abbott et al. 2006) and Santos (Santos and Monteagudo 2012) were held constant (except mutation rate which was selected to be between the two values used by Santos) and other parameters were varied iteratively. The output (tumour size, shape, composition and cell type ratios) was examined for concordance with in vivo tumours. The tumours resemble the classic solid mass tumour structure of a necrotic core with a quiescent rim and proliferating rim (Folkman and Hochberg 1973; Sutherland et al. 1986). The tumours also grow to roughly $2-2.5 \mathrm{~mm}$ before overwhelming the nutrients available and needing their own vasculature (Folkman 1971) and then grow to a maximum of $5.5 \mathrm{~mm}$. All calculations were done using an average cell diameter of $25 \mu \mathrm{m}$ (Tom et al. 1976). The tumours grow to this final size over a period of approximately 2 years, assuming a cell division time of 16-24 h. This is in line with growth times for fast growing tumours, which reach clinically detectable size $(0.2 \mathrm{~cm}$ to $1 \mathrm{~mm})$ within 2 years (McArdle et al. 2005).

\section{Results}

With all hallmarks active, every simulation run produced a tumour using parameters described in Table 1. A "tumour" is classified as a mass in which $99 \%$ or more of its alive cells have at least one mutation. The growth over time for a simulation with all hallmarks available can be seen in Fig. 1.

Figure 1a shows total cell counts throughout growth. Initially healthy cells grow rapidly, however around step 20 they sharply decline. Then, around step 25 cancer cells rapidly start to increase. This corresponds to a sharp increase in angiogenic cells as well as cells that avoid apoptosis, and relatively stable numbers of healthy cells.
Figure $1 \mathrm{c}-\mathrm{h}$ shows the images produced from the same simulation. It can be seen in Fig. 1c that initially healthy cells dominate the clone. Death is occurring, most likely due to random cell death or the initial fast killing of any cancerous cells by apoptosis and the immune system. By $1 d$ we can already see the emergence of different cancer phenotypes. There are three major phenotypes present in the tumour from early on. The center of the tumour also begins to die at $1 \mathrm{~d}$. This is due to a lack of oxygen getting to the center of the tumour (causing necrosis). At $1 \mathrm{~g}$ the outside of the tumour is also dying, as regular cells can go no further as they are outside the growth factor and blood range. In $1 \mathrm{~h}$ we see the tumour is almost entirely cancerous, with a few different phenotypes protruding from the mass. This "fingering morphology", where the border is not smooth but rough, is consistent with other models and histopathological observations (Bello et al. 2004; Anderson et al. 2006; Bellomo and Angelis 2008). It is believed that there are two forms of tumour invasion-either tumour cells outgrow normal tissue and expand as a bulk mass, or they form invasive contingents by intermingling with stromal cells. The fingering morphology is a consequence of this intermingling (Kam et al. 2012). It has been noted that this fingering morphology looks like a crab, from which the word cancer was derived (Kam et al. 2012). The fingering morphology is correlated with harsher microenvironments where only cells with particular phenotypes survive. This behaviour is evident in our model where certain subclones and phenotypes dominate the tumour.

Knocking out some hallmark pairs had very little effect on the growth of the tumour. In fact, knockout pairs SG \& IGI, SG \& AA, SG \& IT, IGI \& AA, IGI \& GU, IGI \& AI, AA \& GU, AA \& AI, IT \& GU and GU \& AI had no significant effect (all $p$ values $>0.05$ using Wilcoxon signed-rank test Bonferroni corrected for multiple comparisons) (see Fig. 2b, c for examples of final simulation image when cancer took over despite hallmark pair knockouts). Other pairs of hallmarks had such a large effect that a cancerous tumour never took over and the simulation ended prematurely as not enough cells survived. The normal cells continued to grow to the edge of the growth factor barrier, and then eventually consumed all of the oxygen in the system and the healthy cells died off. This can be seen in Fig. 2d, e. The following hallmark pairs significantly ( $p=0.0004$ using Wilcoxon signed-rank test, Bonferroni corrected) decreased cancer growth: SG \& A, SG \& GU, SG \& AI, IGI \& A, AA \& A and A \& GU. Other pairs, AA \& IT and A \& AI had a smaller but still significant effect ( $p=0.017$ and $p=0.019$ respectively using Wilcoxon signed-rank test, Bonferroni corrected).

The effect of various hallmark pairs can be seen in Fig. 3. This shows that some hallmark knockouts (A \& GU, IGI \& A, IT \& A, SG \& A, A \& AI, SG \& AI) do not 

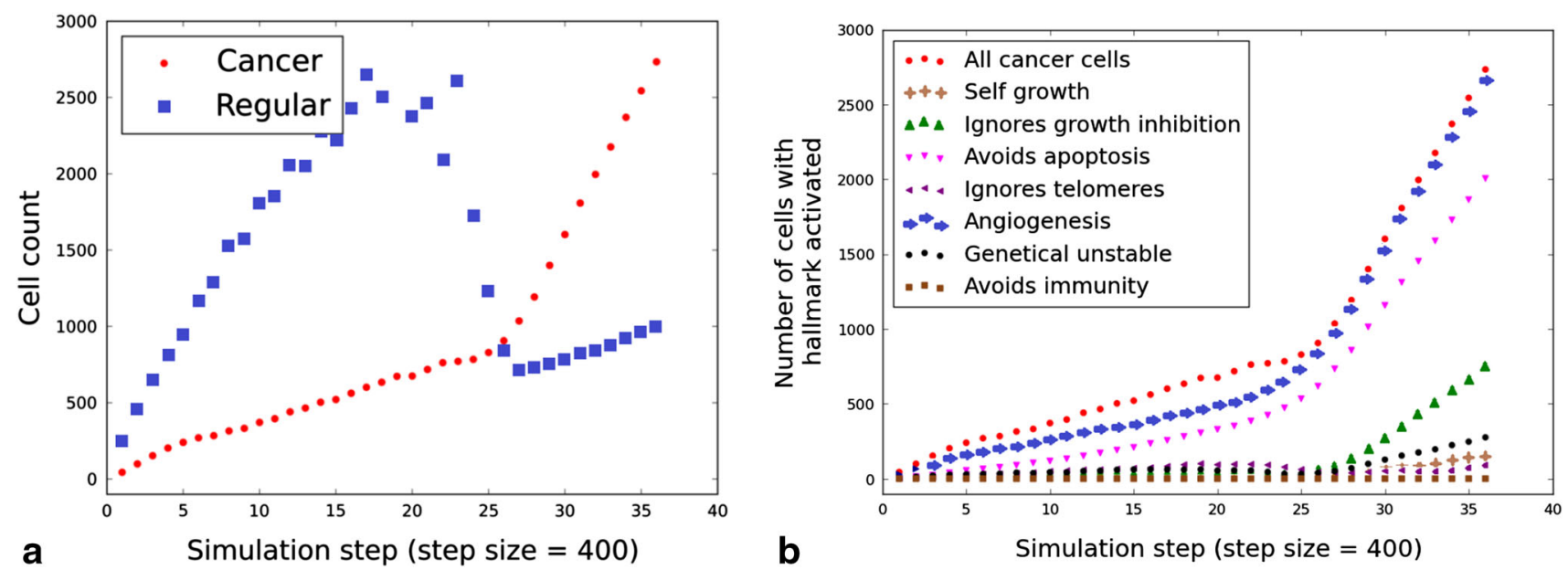
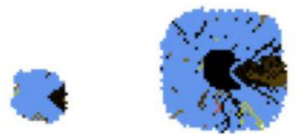

C

d

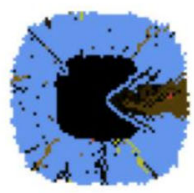

e

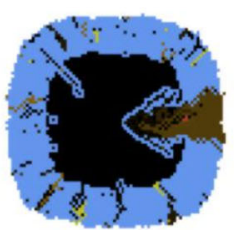

f

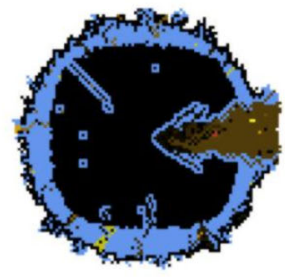

g

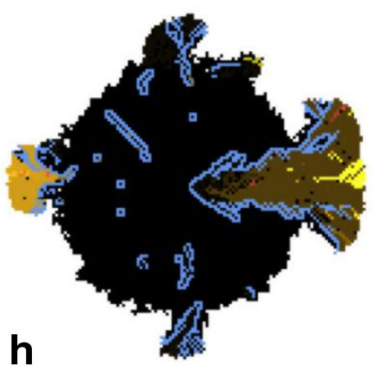

Fig. 1 Total cell count for cancerous, non cancerous, and each hallmark is shown for an entire simulation will all hallmarks available for activation. a Regular versus cancerous cell growth, b each individual hallmark growth and total cancer cell growth. $\mathbf{c}-\mathbf{h}$ Simulation of a colony of cells with all hallmarks available for activation at event steps 1-32. Dead cells are black, healthy cells are blue, all other colours represent some kind of unique cancer phenotype phenotype. Simulation steps: c 1, d 6, e 12, f 18, g 24, h 32. (Color figure online)

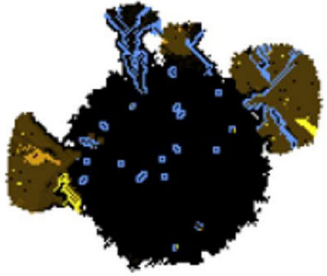

a

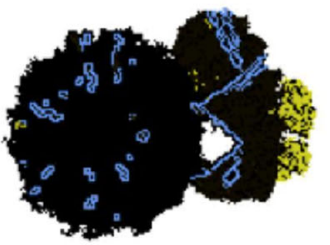

b

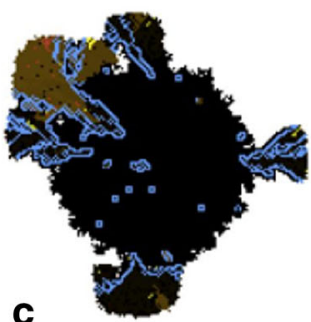

C

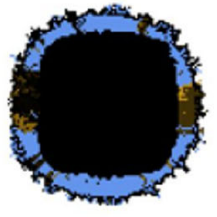

d

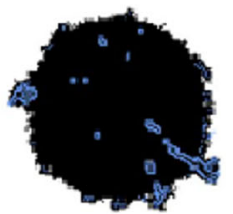

e

Fig. 2 End of simulation images for four different hallmark-knockout pairs. a No hallmarks knocked out, b IGI \& AA, c IGI \& IT, d SG \& A, e IGI \& A

result in a tumour. Cancer growth is fairly consistent across all of the simulations, regardless of knockout, until step 20. Here, all cell populations take a dip however certain simulations show strong growth after this point. The knockout pairs listed above however die off at this point, and these simulations do not result in a cancerous tumour.

Figure 4 shows a histogram of phenotypes that were in the top 10 phenotypes by cell count during the last stage of simulation for 14 unique simulations (knockout pairs that still resulted in a tumour). IDs can be mapped to phenotype using Table 2. While it is obvious that phenotype 0 (dead phenotype) will be present in large numbers in all runs, it is interesting that phenotype 4 and 132 are also present in every run in large numbers-these are cells with the ANGIOGENESIS hallmark activated and no other hallmarks, both alive and dead. Also, by the end of simulation almost all tumours have a large population of dead SELF GROWTH cells and AVOIDS APOPTOSIS cells. It is interesting that alive ANGIOGENESIS cells dominate but dead SELF GROWTH and APOPTOSIS. Also present in the majority of simulations in large numbers are dead cells with IGNORES GROWTH INHIBITION activated and IGNORES TELOMERES activated, as well as alive cells that are both ANGIOGENIC and AVOID APOPTOSIS. 


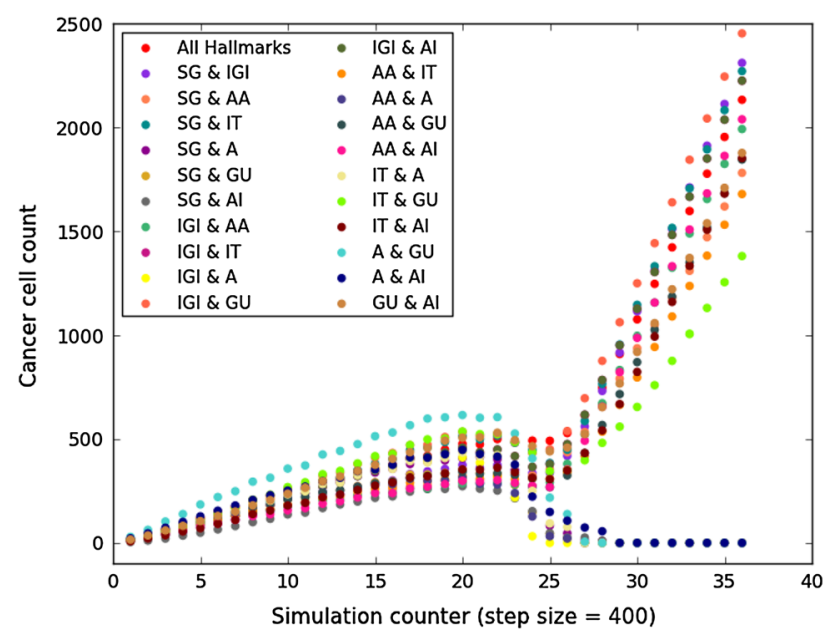

Fig. 3 Total alive cancer cell count every 400 simulation steps is shown. Each hallmark-knockout pair was simulated and run 10 times. The average cell count from these runs was calculated and plotted

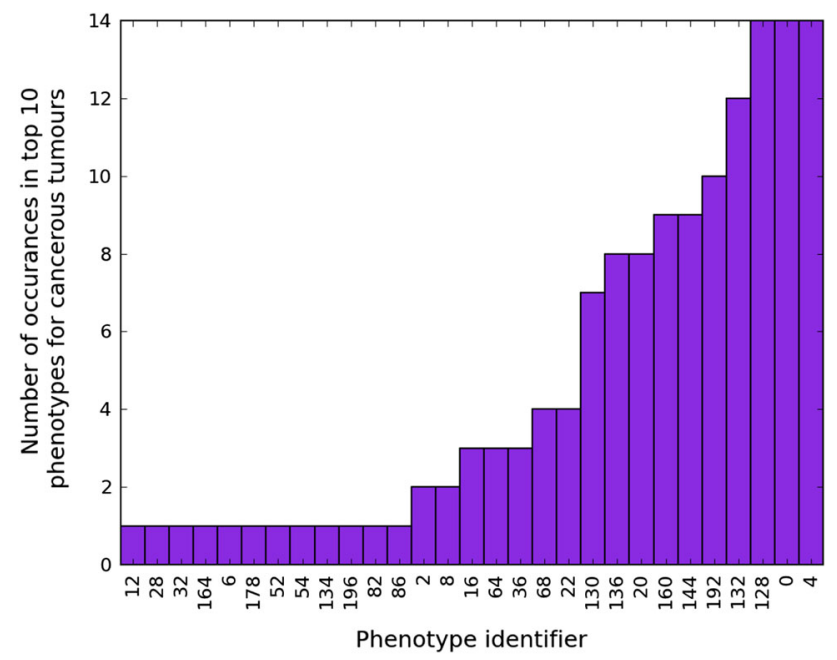

Fig. 4 Each tumour at the end of the simulation had different phenotypes present. The top 10 phenotypes, by total number in the tumour, in each separate simulation were recorded at the end of simulation and totals were plotted

\section{Discussion}

As expected, when all hallmarks are available for activation the tumour grows to the largest extent, presumably as these tumours can take advantage of all hallmarks and the different abilities each confers. It was also expected that knocking out 2 hallmarks would significantly lower the growth of cancer. We hypothesized that certain pairs would perform better than others, and that knocking out hallmarks in pairs could have more than just an additive effect.

Half of all tumours with SELF GROwTH knocked out did not result in a tumour. SG allows a tumour to extend beyond the normal boundary of growth. In areas of the body where growth factor is limited, this would be a very important hallmark. However if a tumour is growing where there is ample growth factor the hallmark may be less effective as a drug target.

All simulations with ANGIOGENESIS knocked out failed to result in a tumour. Similar to SG, ANGIOGENESIS allows a cell to live outside the predefined blood boundary. One reason the ANGIOGENESIS hallmark is more powerful is because it conveys benefit to not just the cell with the mutation, but surrounding cells, as all nearby cells benefit from the new vasculature.

The last hallmark that was knocked out in more than one pair that did not lead to a tumour is GENOME INSTABILITY. Since GENOME INSTABILITY can lead to all of the other mutations being activated more frequently this is understandable.

As evident in Fig. 3, there is a bifurcation in total cell count-either similar to both hallmarks or almost none. This is because cell populations which result in a tumour show almost exponential growth and are not limited by oxygen or space due to acquired mutations. Cell populations that do not result in a tumour are limited by both of these factors, and so eventually almost all cells die as this overpopulation cannot be sustained by the normal vasculature.

It is interesting that of the phenotypes that dominated clones at the end of simulation (shown in Fig. 4), many cells with a single mutation grew quickly but died off. Single mutation phenotypes were largely present, but in dead cells. In contrast, the phenotypes that dominated and were still alive had multiple mutations. While multiple mutations increase the chances of death by the immune system and apoptosis, this suggests it still conveys a very strong advantage overall. This supports the hypothesis that knocking out multiple hallmarks, if you can find the correct pairs, will be better than single target treatments.

Many knockouts did not prevent the tumour from forming. For example, all knockouts that included IGNORING GROWTH INHIBITION still resulted in a tumour, except for one (IGI \& A). In our model IGI allows cells to grow even when there is no space around them, but this only conveys an advantage to internal cells. Cells on the proliferating edge always have space, and therefore removing it does not seem to hurt growth to a significant degree. This could be a limitation of the model as in reality the proliferating rim of a tumour may have space constraints from surrounding tissue.

Other limitations of the model include the fact that ANGIOGENESIS only provides a benefit to itself or cells immediately around it. In addition, it only provides an advantage while the cell is living. In reality angiogenic cells start the creation of blood vessels and those remain even if the cells die. 
Table 2 Phenotype codes and the corresponding hallmarks present in the phenotype

\begin{tabular}{|c|c|}
\hline Code & Hallmarks present \\
\hline 0 & Healthy \\
\hline 128 & Healthy dead \\
\hline 4 & Angiogenesis \\
\hline 132 & Dead, angiogenesis \\
\hline 192 & Dead, self groth \\
\hline 144 & Dead, avoids apoptosis \\
\hline 169 & Dead, ignores growth inhibition, ignores telomers, avoids immunity \\
\hline 29 & Avoids immunity, angiogenesis, ignores telomere, avoids apoptosis \\
\hline 136 & Dead, ignore telomeres \\
\hline 139 & Dead, avoids immunity, genome unstable, ignores telomere \\
\hline 22 & Genome unstable, angiogenesis, avoids apoptosis \\
\hline 68 & Angiogenesis, self grows \\
\hline 36 & Angiogenesis, ignores growth inhibition \\
\hline 64 & Self growth \\
\hline 16 & Avoids apoptosis \\
\hline 8 & Ignores telomeres \\
\hline 2 & Genome unstable \\
\hline 86 & Genome unstable, angiogenesis, avoids apoptosis, self growth \\
\hline 82 & Genome unstable, avoid apoptosis, self growth \\
\hline 196 & Dead, angiogenesis, self grows, dead \\
\hline 134 & Dead, genome unstable, angiogenesis \\
\hline 54 & Genome unstable, angiogenesis, avoids apoptosis, ignores growth inhibition \\
\hline 52 & Angiogenesis, avoids apoptosis, ignores growth inhibition \\
\hline 178 & Dead, genome unstable, avoids apoptosis, ignores growth inhibition \\
\hline 6 & Genome unstable, angiogenesis \\
\hline 164 & Dead, angiogenesis, ignores growth inhibition \\
\hline 32 & Ignores growth inhibition \\
\hline 28 & Angiogenesis, ignores telomere, avoids apoptosis \\
\hline 12 & Angiogenesis, ignores telomere \\
\hline
\end{tabular}

\section{Conclusions}

We have modelled the impact of cancer hallmarks, as proposed by Hanahan and Weinberg, on early tumour growth using a cellular automaton model of cancer cells and lattice Boltzmann methods for two phase fluids (oxygen in the blood, which is more physiologically relevant than the more common choice of modelling just oxygen or blood alone, as oxygen is a fluid transported in blood) (Hanahan and Weinberg 2000, 2011). Our results show that knocking out pairs of hallmarks does not necessarily have an additive effect. Santos et al. found that AVOIDING APOPTOSIS and IGNORING GROWTH INHIBITION were the most critical hallmarks independently when cells had a high rate of mutation, and they also found that IGNORING TELOMERES and SELF GROWTH had a small impact (Santos and Monteagudo 2012). Looking at the impact of knocking out both AVoIDING APOPTOSIS and (IGNORING GROWTH INHIBITION), we did not see a significant decrease in tumour growth. This is interesting as it is not what would be expected from the findings of knocking out singular hallmarks if one assumes linear combination of knockout effects.

We found that knocking out the ability for a cancer cell to SELF GROW and AVOID IMMUNE SYSTEM, as well as SELF GROW and be GENETICALLY UNSTABLE, prevent a tumour from growing. Neither SELF GROWTH nor GENETIC INSTABILITY had a great effect in the simulations done by Santos et al. (the immune system was not modelled in this work) however in combination they had a strong and significant effect. This supports our hypothesis that knowing the impact of individual hallmarks, which can be extended to individual drugs, does not necessarily give insight into the impact of combining those knockouts and drugs.

Lastly, we found that knocking out the ability for cells to INDUCE ANGIOGENESIS combined with any other hallmark prevented tumour growth. Research has been done into anti-angiogenesis drugs however the conclusions were not always positive. Patients still died from small tumours throughout the body as opposed to one large tumour, which was seen without the drugs (Ebos et al. 2009). It is 
hypothesized that without angiogenesis, other factors became important, such as metastasis. Perhaps the key is preventing cells from inducing angiogenesis and limiting other cancerous abilities. Currently trials are underway to test pairing anti-angiogenesis drugs with various chemotherapy drugs for multiple types of cancer including breast, colon and medulloblastoma (Chinese Academy of Medical Sciences 2014; International breast cancer study group 2014; Hoffmann-La 2014; Medical University of Vienna 2014; Hellenic Oncology Research Group 2014)_in other words, combination therapy trials.

We have found that the effect of knocking out cancer hallmarks in pairs can have varying levels of success. This suggests that clinical research should be done into combination drug treatment as not all drugs that are strong individually will necessarily be strong in combination. Since cancer treatments can be physically and emotionally challenging for patients, knowing in advance what combinations will not be successful could greatly enhance the quality of life of people undergoing cancer treatment.

\section{References}

Abbott R, Forrest S, Pienta K (2006) Simulating the hallmarks of cancer. Artif Life 4(12):617-34

Anderson ARA, Quaranta V (2008) Integrative mathematical oncology. Nat Rev Cancer 8(3):227-234. doi:10.1038/nrc2329, http:// www.ncbi.nlm.nih.gov/pubmed/18273038

Anderson ARA, Weaver AM, Cummings PT, Quaranta V (2006) Tumor morphology and phenotypic evolution driven by selective pressure from the microenvironment. Cell 127(5):905-915. doi:10.1016/j.cell.2006.09.042, http://www.ncbi.nlm.nih.gov/ pubmed/17129778

Anderson ARA, Rejniak KA, Gerlee P, Quaranta V (2008) Modelling of cancer growth, evolution and invasion: bridging scales and models. Math Model Nat Phenom 2(3):1-29. doi:10.1051/mmnp:2007001, http://www.mmnp-journal.org/10.1051/mmnp:2007001

Artandi SE, DePinho RA (2010) Telomeres and telomerase in cancer. Carcinogenesis 31(1):9-18

Basanta D, Ribba B, Watkin E, You B, Deutsch A (2011) Computational analysis of the influence of the microenvironment on carcinogenesis. Math Biosci 229(1):22-29. doi:10.1016/j. mbs.2010.10.005, http://www.sciencedirect.com/science/article/ pii/S0025556410001616

Bello L, Lucini V, Costa F, Pluderi M, Giussani C, Acerbi F, Carrabba G, Pannacci M, Caronzolo D, Grosso S, Shinkaruk S, Colleoni F, Canron X, Tomei G, Deleris G, Bikfalvi A (2004) Combinatorial administration of molecules that simultaneously inhibit angiogenesis and invasion leads to increased therapeutic efficacy in mouse models of malignant glioma combinatorial administration of molecules that simultaneously inhibit angiogenesis. Clin Cancer Res 10(13):4527-4537

Bellomo N, De Angelis E (2008) Selected topics in cancer modeling: genesis, evolution, immune competition, and therapy. Springer, Berlin

Bentley K, Bates P, Gerhardt H (2008) Artificial life as cancer research: embodied agent modelling of blood vessel growth in tumours. In: Proceedings of artifical life XI
Berdasco M, Esteller M (2010) Aberrant epigenetic landscape in cancer: how cellular identity goes awry. Dev Cell 19(5):698-711

Bhatnagar E, Gross E, Krook M (1954) A model for collision processes in gases. I. Small amplitude processes in charged and neutral one-component systems. Phys Rev 94(3):511

Chen S, Doolen GD (1998) Lattice boltzmann method for fluid flows. Annu Rev Fluid Mech 30:329-64

Chinese Academy of Medical Sciences (2014) Efficacy and safety study of recombinant endostatic combined with chemotherapy to treat advanced colorectal cancer. ClinicalTrials.gov Bethesda (MD): National Library of Medicine (US)

Detjen KM, Rieke S, Deters A, Schulz P, Rexin A, Vollmer S, Hauff P, Wiedenmann B, Pavel M, Scholz A (2010) Angiopoietin-2 promotes disease progression of neuroendocrine tumors. Clin Cancer Res 16(2):420-429

Ebos JML, Lee CR, Cruz-Munoz W, Bjarnason Ga, Christensen JG, Kerbel RS (2009) Accelerated metastasis after short-term treatment with a potent inhibitor of tumor angiogenesis. Cancer Cell 15(3):232-239. doi:10.1016/j.ccr.2009.01.021, http://www. ncbi.nlm.nih.gov/pubmed/19249681

Erbertseder K, Reichold J, Flemisch B, Jenny P, Helmig R (2012) A coupled discrete/continuum model for cancer-therapeutic transport in the lung. PloS One 7(3)

Evan G, Littlewood T (1998) A matter of life and cell death. Science 281(5381):1317-1322

Ferlay J, Shin H, Bray F, Forman D, Mathers C, Parkin D (2008) Globocan2008 cancer incidence and mortality worldwide: Iarc cancerbase no. 10

Folkman J (1971) Tumor angiogenesis: therapeutic implications. Nat Engl J Med 285:L529-L533

Folkman J, Hochberg M (1973) Self-regulation of growth in three dimensions. J Exp Med 138(4):745-753

Gerlee P, Anderson ARA (2007) An evolutionary hybrid cellular automaton model of solid tumour growth. J Theor Biol 246(4):583-603. doi:10.1016/j.jtbi.2007.01.027, http://www. ncbi.nlm.nih.gov/pmc/articles/PMC2652069/?tool=pmcentrez

Gerlee P, Anderson ARA (2008) A hybrid cellular automaton model of clonal evolution in cancer: the emergence of the glycolytic phenotype. J Theor Biol 250:705-722

Gerlee P, Anderson ARA (2009) Evolution of cell motility in an individual-based model of tumour growth. $\mathrm{J}$ Theor Biol 259(1):67-83. doi:10.1016/j.jtbi.2009.03.005

Gevertz JL, Gillies GT, Torquato S (2008) Simulating tumor growth in confined heterogeneous environments. Phys Biol 5(3):036010. doi:10.1088/1478-3975/5/3/036010, http://www.ncbi.nlm.nih. gov/pubmed/18824788

Hanahan D, Weinberg R (2000) The hallmarks of cancer. Cell 100(1):57-70

Hanahan D, Weinberg R (2011) Hallmarks of cancer: the next generation. Cell 144(5):646-674

Hellenic Oncology Research Group (2014) Paclitaxel plus bevacizumab for older patients with breast cancer. ClinicalTrials.gov Bethesda (MD): National Library of Medicine (US)

Henderson E, Samaha R (1969) Evidence that drugs in multiple combinations have materially advanced the treatment of human malignancies. Cancer Res 29(12):2272-2280

Hirata Y, Bruchovsky N, Aihara K (2010) Development of a mathematical model that predicts the outcome of hormone therapy for prostate cancer. J Theor Biol 264(2):517-527. doi:10.1016/j.jtbi.2010.02.027, http://www.ncbi.nlm.nih.gov/ pubmed/20176032

Hoffmann-La Roche (2014) A study of avastin (bevacizumab) in combination with chemotherapy in patients with breast cancer progressing after first-line therapy with avastin and chemotherapy (tania). ClinicalTrials.gov Bethesda (MD): National Library of Medicine (US) 
International breast cancer study group (2014) Combination chemotherapy after surgery in treating patients with stage I, stage II or stage III breast cancer. ClinicalTrials.gov Bethesda (MD): National Library of Medicine (US)

Jones PA, Baylin SB (2007) The epigenomics of cancer. Cell 128(4):683-692

Junttila MR, Evan GI (2009) p53 — a Jack of all trades but master of none. Nat Rev Cancer 9(11):821-829

Kam Y, Rejniak KA, Anderson ARA (2012) Cellular modeling of cancer invasion: integration of in silico and in vitro approaches. J Cell Physiol 227(2):431-438. doi:10.1002/jcp.22766, http:// www.ncbi.nlm.nih.gov/pubmed/21465465

Kim R, Emi M, Tanabe K (2007) Cancer immunoediting from immune surveillance to immune escape. Immunology 121(1):1-14

Korkola J, Gray JW (2010) Breast cancer genomes-form and function. Curr Opin Genet Dev 20(1):4-14

Lloyd BA, Szczerba D, Rudin M, Székely G (2008) A computational framework for modelling solid tumour growth. Philos Trans Ser A Math Phys Eng Sci 366(1879):3301-3318. doi:10.1098/rsta. 2008.0092, http://www.ncbi.nlm.nih.gov/pubmed/18593664

Lowe SW, Cepero E, Evan G (2004) Intrinsic tumour suppression. Nature 432(7015):307-315

Macklin P, Edgerton ME, Thompson A, Cristini V (2012) Patientcalibrated agent-based modelling of ductal carcinoma in situ (DCIS) I: model formulation and analysis. J Theor Biol 301:122-140

Maley C, Forrest S (2000) Exploring the relationship between neutral and selective mutations in cancer. Artif Life 6(4):325-345

McArdle WD, Katch FI, Katch VL (2005) Essentials of exercise physiology, 3rd edn. Lippincott Williams \& Wilkins, Philadelphia

Medical University of Vienna (2014) Metronomic and targeted antiangiogenesis therapy for children with recurrent/progressive medulloblastoma (memmat). ClinicalTrials.gov Bethesda (MD): National Library of Medicine (US)

Monteagudo A, Santos J (2012) A cellular automaton model for tumor growth simulation. Adv Intell Soft Comput 154:147-155

Monteagudo A, Santos J (2013) Cancer stem cell modeling using a cellular automaton. IWINAC LNCS 7931:21-31

Monteagudo A, Santos J (2014) Studying the capability of different cancer hallmarks to initiate tumor growth using a cellular automaton simulation. Application in a cancer stem cell context. Biosystems 115:46-58

Olive KP, Jacobetz MA, Davidson CJ, Gopinathan A, McIntyre D, Honess D, Madhu B, Goldgraben MA, Caldwell ME, Allard D et al (2009) Inhibition of hedgehog signaling enhances delivery of chemotherapy in a mouse model of pancreatic cancer. Science 324(5933):1457-1461

Orlandini E, Swift MR, Yeomans J (1995) A lattice boltzmann model of binary-fluid mixtures. EPL (Europhysics Letters) 32(6):463

Perona R (2006) Cell signalling: growth factors and tyrosine kinase receptors. Clin Transl Oncol 8(2):77-82

Raica M, Cimpean AM, Ribatti D (2009) Angiogenesis in premalignant conditions. Eur J Cancer 45(11):1924-1934

Ramis-Conde I, Chaplain M AJ, Anderson ARA (2008) Mathematical modelling of cancer cell invasion of tissue. Math Comput Model 47(5-6):533-545. doi:10.1016/j.mcm.2007.02.034, http://linkin ghub.elsevier.com/retrieve/pii/S0895717707001823

Rejniak KA, Anderson ARA (2011) Hybrid models of tumor growth. Wiley Interdiscip Rev Syst Biol Med 3(1):115-125. doi:10.1002/ wsbm.102, http://www.ncbi.nlm.nih.gov/pmc/articles/PMC30 57876/?tool=pmcentrez

Rejniak KA, Anderson ARA (2012) State of the art in computational modelling of cancer. Math Med Biol 29(1):1-2. doi:10.1093/ imammb/dqr029, http://www.ncbi.nlm.nih.gov/pubmed/22200587

Ribba B, Alarcón T, Marron K, Maini PK, Agur Z (2004) The use of hybrid cellular automaton models for improving cancer therapy. In: Sloot PMA, Chopard B, Hoekstra AG (eds) Cellular automata. Lecture notes in computer science, vol 3305. Springer, Hiedelberg, pp 444-453

Santos J, Monteagudo Á (2012) Study of cancer hallmarks relevance using a cellular automaton tumor growth model. In: Parallel problem solving from nature-PPSN XII, pp 489-499

Sener S, Fremgen A, Menck H, Winchester D (1999) Pancreatic cancer: a report of treatment and survival trends for 100,313 patients diagnosed from 1985-1995, using the national cancer database. J Am Coll Surg 189(1):1-7

Shrestha SMB, Joldes GR, Wittek A, Miller K (2013) Cellular automata coupled with steady-state nutrient solution permit simulation of large-scale growth of tumours. Int $\mathrm{J}$ Numer Methods Biomed Eng 29(4):542-559

Spencer SL, Berryman MJ, Garcia JA, Abbott D (2004) An ordinary differential equation model for the multistep transformation to cancer. J Theor Biol 231:515-524

Succi S (2001) The lattice Boltzmann equation for fluid dynamics and beyond. Oxford University Press, Oxford

Sun X, Zhang L, Tan H, Bao J, Strouthos C, Zhou X (2012) Multiscale agent-based brain cancer modeling and prediction of TKI treatment response: incorporating EGFR signaling pathway and angiogenesis. BMC Bioinform 13(1):218. doi:10.1186/14712105-13-218, http://www.biomedcentral.com/1471-2105/13/218

Sutherland R, Sordat B, Bamat J, Gabbert H, Bourrat B, MuellerKlieser W (1986) Oxygenation and differentiation in multicellular spheroids of human colon carcinoma. Cancer Res 46(10):5320-5329

Swift M, Orlandini E, Osborn W, Yeomans J (1996) Lattice Boltzmann simulations of liquid-gas and binary fluid systems. Phys Rev E 54(5):5041-5052. http://www.ncbi.nlm.nih.gov/ pubmed/9965683

Teng MW, Swann JB, Koebel CM, Schreiber RD, Smyth MJ (2008) Immune-mediated dormancy: an equilibrium with cancer. J Leukoc Biol 84(4):988-993

Tom BH, Rutzky LP, Jakstys MM, Oyasu R, Kaye CI, Kahan BD (1976) Human colonic adenocarcinoma cells. In Vitro 12(3):180-191

Vajdic CM, van Leeuwen MT (2009) Cancer incidence and risk factors after solid organ transplantation. Int $\mathrm{J}$ Cancer 125(8):1747-1754

Valvano JW, Cochran JR, Diller KR (1985) Thermal conductivity and diffusivity of biomaterials measured with self heated thermistors. Int J Thermophys 6(3):301-311

Witsch E, Sela M, Yarden Y (2010) Roles for growth factors in cancer progression. Physiology 25(2):85-101

World Health Organization (2008) The global burden of disease: 2004 update. http://www.who.int/healthinfo/global_burden_disease/ 2004_report_update/en/ 